\title{
EchoGéo
}

18 | 2011

Varia

\section{Les risques en milieu urbain : éléments de réflexion}

\section{Pascale Metzger et Robert D'Ercole}

\section{(2) OpenEdition}

Journals

Édition électronique

URL : https://journals.openedition.org/echogeo/12640

DOI : 10.4000/echogeo. 12640

ISSN : 1963-1197

\section{Éditeur}

Pôle de recherche pour l'organisation et la diffusion de l'information géographique (CNRS UMR 8586)

\section{Référence électronique}

Pascale Metzger et Robert D'Ercole, «Les risques en milieu urbain : éléments de réflexion », EchoGéo [En ligne], 18| 2011, mis en ligne le 06 décembre 2011, consulté le 01 août 2021. URL : http:// journals.openedition.org/echogeo/12640; DOI : https://doi.org/10.4000/echogeo.12640

Ce document a été généré automatiquement le 1 août 2021

EchoGéo est mis à disposition selon les termes de la licence Creative Commons Attribution - Pas d'Utilisation Commerciale - Pas de Modification 4.0 International (CC BY-NC-ND) 


\title{
Les risques en milieu urbain : éléments de réflexion
}

\author{
Pascale Metzger et Robert D'Ercole
}

$1 \quad$ L'objectif de ce texte est de contribuer au débat sur la question des risques, et plus spécifiquement sur les risques en milieu urbain, en décalant un peu le questionnement habituel. L'option prise est de souligner les problèmes conceptuels posés par l'approche des risques, notamment en géographie. Il faut voir ce texte comme l'introduction à un débat sur l'approche des risques par nos disciplines, principalement par la géographie, qui en même temps ouvre quelques pistes de réflexion sur l'articulation des différentes problématiques du risque dans les politiques publiques ${ }^{1}$.

2 De quoi parle $t$-on quand on parle de risques en milieu urbain? Nous allons d'abord parler des risques vus par le sens commun, en toute généralité, démarche nécessaire dans le sens où cette façon d'introduire les risques interpelle la démarche scientifique. Dans une deuxième étape, seront abordés les risques comme objet scientifique, c'est-àdire comment la démarche scientifique approche et construit cet objet particulier du monde social. Chemin faisant, on abordera à plusieurs reprises les risques comme objets de politiques publiques, et les risques impliqués directement ou indirectement dans les politiques publiques. Mais c'est principalement le deuxième point qui sera développé, autrement dit l'approche scientifique des risques.

Cette présentation essaie paradoxalement d'aborder la question des risques en toute généralité, mais aussi dans toute sa complexité, dans ses liens avec les grands débats qui agitent le monde, dans les articulations et distances entre les risques vus par le sens commun et ceux étudiés par la géographie.

4 Les conséquences dévastatrices du séisme d'Haïti de 2010 ont fait la démonstration magistrale de ce qu'est la vulnérabilité, de l'importance de la vulnérabilité dans les risques, et de la dimension majeure de la pauvreté dans cette vulnérabilité. Aussi, les politiques de prévention basées sur une conception étroite des risques principalement basée sur l'aléa ne peuvent avoir qu'une efficacité très limitée. La prévention des risques sismiques se situe largement hors du champ du risque sismique, dans le développement social et économique, la démocratie et la justice, dans des politiques du 
logement et d'aménagement du territoire, dans des politiques de santé et d'équipements. Le séisme du Chili cette même année 2010 a fait une démonstration supplémentaire, celle de l'importance des capacités à gérer les situations de crise dans ce qui fait le risque et plus précisément la vulnérabilité. L'actualité de ces derniers mois vient renforcer les propos tenus. Les vicissitudes des volcans irlandais, le séisme et le tsunami du Japon en 2011 montrent l'importance des enchaînements et la vitesse des mécanismes de propagation du risque qui échappent à tout contrôle dans tous les domaines de la société, et au-delà de la prévention, la difficile question de la préparation aux situations de crise.

5 La gestion des situations d'urgence et de crise doit devenir un véritable objet de recherche, non seulement des politistes et sociologues, mais aussi des géographes (D'Ercole \& Metzger, 2009), dans le but de participer à la construction de connaissances réellement utiles à la réduction de la vulnérabilité des sociétés et des territoires du Sud comme du Nord.

\section{En première approche : les risques dans le sens commun}

$6 \quad$ Il est assez habituel lorsqu'on procède à des enquêtes auprès de la population sur les risques d'origine naturelle de se trouver face à des réponses décalées sur le risque, portant en particulier sur la délinquance vécue au quotidien. D'une certaine manière, en première instance, la recherche scientifique sur les risques porte souvent sur un risque qui ne correspond pas au point de vue des gens.

7 En première approche, quand on parle des risques en milieu urbain, dans le sens commun viennent d'abord les inquiétudes posées par la délinquance urbaine, les problèmes sociaux, ou les accidents de la route par exemple. Le terme risque est utilisé à chaque fois qu'il y a possibilité de perdre quelque chose, pour un individu, une famille, une ville, un territoire, une entreprise, une société, un pays, une organisation sociale quelle qu'elle soit.

Pour donner quelques exemples, relevant de différentes échelles, on peut citer :

- Pour un individu ou une famille, il existe le risque de perdre son travail, d'avoir un problème de santé, un accident de voiture, de se faire agresser, de perdre la vie, mais aussi, le cas échéant, d'avoir sa maison inondée, détruite par un séisme, emportée par un cyclone etc.

- Pour un quartier, on pourra avoir, par exemple, le risque d'une coupure de l'approvisionnement en eau, de voir fermer un service public, d'être socialement stigmatisé, de voir s'implanter une entreprise dangereuse, mais aussi, éventuellement, d'être inondé ou affecté par un risque d'origine naturelle.

- Pour une ville, on peut noter des risques d'un autre ordre, comme celui de perdre un rôle régional ou national, de voir fermer une entreprise importante, un tribunal, un hôpital, une gare ou un aéroport, d'être inondée, affectée par un séisme ou une éruption volcanique.

- Pour un pays aussi on va parler de risques, le fameux « risque pays » des économistes, un risque de guerre ou d'instabilité politique, le risque de se voir paralysé par des mouvements sociaux, le risque de terrorisme, le risque de perdre un rôle important sur la scène internationale... mais aussi d'être affecté par le changement climatique, de subir un séisme, un cyclone, une crise économique ou financière.... 

la problématique des risques, puisque toutes les inquiétudes environnementales portent finalement sur l'existence de risques, leurs conséquences et la façon d'y faire face. Risques sur la biodiversité, sur la santé humaine, sur la préservation d'espaces, sur les ressources en eau, sur la qualité de l'air, l'épuisement des sols, les risques que présentent les nouvelles technologies, les risques de changement climatique etc. Il y a donc une parenté directe qui reste à expliciter et à formaliser entre la problématique des risques et la problématique environnementale (Metzger, 1999). Cependant, les inquiétudes environnementales introduisent incontestablement une nouvelle dimension du risque en impliquant le temps de différentes manières (Ewald, 1997) : il y a les risques d'aujourd'hui, mais fondamentalement, dans la question environnementales, il y a les risques qui pèsent sur les générations futures; il y a les risques qui se matérialisent brutalement qu'on identifie assez clairement sous la forme d'évènements possibles (une agression, une inondation...), mais aussi les risques qui se construisent progressivement sur le temps long comme le changement climatique ou la pollution de l'eau, des sols, etc.

12 La formalisation et la réponse à tous ces risques sont d'abord venues des philosophes, avec des notions telles que le principe de responsabilité et le principe de précaution (Habermas, 1973; Jonas, 1990). Ces notions font maintenant partie du vocabulaire commun, elles sont entrées dans les discours habituels, dans le droit et les politiques publiques, mais aussi dans l'espace public, du local à l'international (Gilbert, 1997). Ainsi dans la sphère politique se discute, se débat, explicitement ou non, à travers la problématique environnementale, la question des risques, de leur existence, de leurs causes, de leurs conséquences et de la façon de les diminuer, de les éviter, d'y faire face... L'incertitude portée par les «nouveaux risques» questionne la science positiviste et renvoie à des systèmes de croyance (Godard, 1997 ; Godard, Henry, Lagadec et Michel-Kerjan, 2002 ; Theys et Kalaora, 1992).

13 On voit immédiatement que les risques, puisqu'ils sont largement construits par un point de vue social et territorial (ce que l'on peut perdre), se posent presque systématiquement en terme de contradictions : contradictions entre différents risques, qui traduisent et expriment des conflits d'intérêts, contradictions entre intérêt collectif et intérêt individuel, contradictions entre groupes sociaux, entre territoires, entre systèmes de valeurs (Borraz, 2005).

14 Pour reprendre quelques exemples rapides, le syndrome Nimby (Not In My Back Yard) exprime le conflit entre intérêt individuel et intérêt collectif, mais aussi et plus certainement, entre lieux qui sont susceptibles de supporter le risque, entre types de risque, entre d'une part le risque collectif, diffus et plus ou moins lointain de la pollution de l'eau et des sols en l'absence de traitement des eaux usées par exemple et 
d'autre part les nuisances et risques immédiats et localisés liés à la présence des stations d'épurations.

Les risques expriment donc aussi des conflits entre territoires et entre échelles de territoire. Ainsi, l'urbanisation de secteurs considérés comme dangereux, modalité habituelle d'accès au sol urbain des populations les plus pauvres des villes du Sud en l'absence de politique du logement, va augmenter les risques, la déforestation étant susceptible d'augmenter le ruissellement et de déstabiliser des terrains, d'où les risques d'inondation ou de glissement de terrain en aval (Chaline et Dubois Maury, 1994).

L'exemple d' $\mathrm{AZF}^{2}$, emblématique des risques technologiques, montre clairement que les risques posés par cette usine sont de plusieurs ordres, différents en fonction des positions sociales, acteurs impliqués, échelles concernées: on voit distinctement s'opposer le risque à l'échelle de l'individu de perdre son emploi et celui, à l'échelle de la ville, de perdre une activité économique majeure, au risque local de perdre la vie, la santé, son logement, dans une explosion.

17 Autre exemple d'opposition entre risques qui se situent sur différents plans, à différentes échelles spatiales ou de temps, le risque alimentaire ou sanitaire s'oppose souvent au risque de contamination par les pesticides: pour lutter contre le risque alimentaire ou sanitaire, on va répandre des pesticides qui vont provoquer un autre type de risque sur une autre échelle de temps. De la même façon, le risque nucléaire va entrer en contradiction avec le risque de réchauffement climatique, les choix opérés déplaçant à la fois le type de risques, de territoires et de populations concernées... L'énergie nucléaire qui était perçue comme un risque majeur dans les années 70-80 devient une solution dans les années 2000.

On voit ainsi que faire face à ces risques renvoie à des formulations variées qui appellent des réponses àdifférentes échelles: des comportements individuels, des stratégies collectives, des politiques urbaines, des politiques publiques nationales, des actions internationales ... Ainsi, la gestion des risques correspond bien à un arbitrage entre différents types de risques (Dourlens \& Vidal Naquet, 1992 ; Barraqué, 1994).

Globalement les risques renvoient à l'insécurité (sociale, sanitaire, économique, politique, physique). En face des risques, dans le sens commun, on a la sécurité et tout système d'assurance, qu'il soit individuel ou collectif. Donc on a aussi l'Etat, garant de la sécurité des citoyens, et toutes les politiques d'assistance, la sécurité sociale, l'assurance chômage en plus des politiques explicites de prévention des risques (Borraz, 2008). Mais on voit aussi dans la question des risques se profiler les politiques économiques. Par exemple, le développement de cultures d'exportation destinées avant tout à produire des devises voit s'opposer un risque économique ou financier à l'échelle nationale, voire internationale, à des risques sociaux ou environnementaux à une échelle beaucoup plus locale dans les territoires transformés en zone de monoculture. Autre exemple, en écho à ce qui a été dit précédemment, le choix du nucléaire dans le cadre d'une politique nationale d'indépendance énergétique va concrètement et localement avoir des implications en termes de risque.

Ainsi des politiques très lointaines par rapport à la problématique du risque, des politiques nationales qui apparemment n'ont rien à voir avec le risque, et en tout cas sont rarement prises en compte dans l'analyse des risques, vont de fait arbitrer entre des types de risques, entre des temps du risque, entre les lieux du risque (Pigeon, 2005 ; Gilbert, 2007 ; Cartier, Vinet, Gaillard, 2009). 
Ce panorama du sens commun des risques met en évidence la diversité des risques et montre que la question des échelles et du point de vue territorial et social joue un rôle dans ce qui fait le risque, dans ce qu'est le risque, dans ce que sont les politiques publiques, qu'elles soient ou non affichées comme politiques de gestion du risque. Le risque est donc bien une affaire de géographes et de sciences sociales. Il s'agit aussi d'insister sur le fait que derrière la formulation de l'existence de risques il y a nécessairement des conflits, des intérêts contradictoires, et derrière les politiques publiques, il y a souvent, implicitement, des arbitrages entre risques, qui opèrent un déplacement social ou spatial ou temporel du risque. Il s'agit donc bien à la fois d'un problème politique, et d'un objet de politiques publiques (Borraz, 2008). Ainsi ce qui fait les risques dans une société, ce qui aboutit à des risques très concrets sur un territoire, a des racines très profondes qui peuvent se situer très loin du territoire concerné, dans de lointaines politiques publiques, dans des décisions et des choix qui se jouent ailleurs et sur un tout autre registre. Tous ces éléments laissent entrevoir les problèmes posés par la gestion démocratique des risques, par la gouvernance des risques pour reprendre un terme à la mode.

\section{Le risque comme objet scientifique}

Face à cette diversité, à cette complexité du risque, bien présentes dans le monde social, comment la science ou plutôt les disciplines scientifiques, et en particulier la géographie, ont-elles approché les risques? En première approche, on peut constater que la problématique des risques est éclatée en de multiples spécialités, construites principalement autour de ce qui est identifié par ces spécialités comme étant la cause du risque, et c'est cette cause désignée du risque qui va construire un type de risque, dans une démarche admirablement déterministe ${ }^{3}$.

Les risques naturels (devenus «d'origine naturelle») sont appréhendés par les disciplines spécialistes du risque inondation, de crues, de glissement de terrain, des risques volcaniques, sismiques, d'effondrements etc. Bref, ce sont des risques qui sont définis d'abord par leur origine dans un processus physique et naturel, dont on va cependant reconnaître qu'il est plus ou moins altéré par des phénomènes anthropiques, en particulier par l'urbanisation et la déforestation.

Les risques anthropiques, en particulier les risques industriels et technologiques liés aux installations dangereuses, les risques dus au transport de matériels dangereux, les risques automobiles..., ont aussi leurs spécialistes. Là encore, la spécialité est dans la cause, l'origine identifiée du risque immédiate et localisée (les produits dangereux, les usines dangereuses).

Les risques sociaux, type risques politiques, risque de guerre, la délinquance etc., c'est la société, l'organisation sociale et politique qui est la cause du risque, c'est donc un champ abordé par les différentes disciplines des sciences sociales.

Les risques sanitaires semblent une espèce à part dans la grande problématique des risques, car ils sont construits non pas sur la cause du risque mais sur ce qu'on ne veut pas perdre, sur ce qu'on veut protéger, c'est-à-dire sur la santé : c'est tout ce qui peut affecter la santé, qu'elle soit humaine ou animale, qui est approché par les risques sanitaires, quelles qu'en soient les causes. 
Pour les risques environnementaux, la situation est moins claire: on a surtout des approches dans lesquels l'environnement (le milieu dans lequel on vit) devient un risque, du fait par exemple de la pollution de l'eau, des sols, de l'air, ou la déforestation. On serait donc dans le risque défini par la cause, découpé par les objets des disciplines ; mais on pourrait également considérer que c'est l'environnement qui est au cœur des risques environnementaux, qu'il s'agit de préserver l'environnement, auquel cas les risques environnementaux seraient tous les risques qui pèsent sur l'environnement.

Ce découpage du champ des risques renvoie le plus souvent à des disciplines des sciences de la terre, en particulier pour tout ce qui concerne les risques d'origine naturelle qui sont les risques les plus souvent abordés par la géographie. C'est essentiellement l'approche scientifique des risques d'origine naturelle dont il sera question ici.

\section{Le concept de risque : déficit conceptuel}

Com dogme pourrait-on dire, est $:$ risque = aléa $\mathrm{x}$ vulnérabilité (Pigeon, 2003).

Ce paradigme "aléa $x$ vulnérabilité » est très puissant car il est ancré, non seulement dans les démarches scientifiques, mais aussi dans les outils concrets de gestion et de prévention des risques mis en place par les politiques publiques, dans les pays du nord comme du sud. La plupart des plans de prévention concernent un seul aléa, et commencent par la "cartographie des risques» c'est-à-dire de l'aléa. Il y a une conséquence directe à cette approche : les politiques publiques sont le plus souvent des politiques d'évitement ou de contention de l'aléa.

31 Le paradigme "aléa x vulnérabilité », dans les analyses de risques d'origine naturelle, renvoie directement à l'opposition nature-culture pourtant très discutée (Pigeon, 2005). On ne sait pas grand-chose du croisement entre l'aléa et la vulnérabilité, à part la conjonction spatiale des deux. Cette dichotomie entre aléa et vulnérabilité opère incontestablement une division du travail entre sciences «dures » qui se chargent de l'aléa et sciences sociales qui analysent la vulnérabilité, alors même que tout le monde revendique la nécessaire pluridisciplinarité pour approcher les risques. Cette division est directement reproduite dans la bipolarisation classique de la géographie entre ses deux composantes majeures que sont la géographie physique et la géographie sociale.

L'approche des risques se fait donc à travers ces deux piliers, mais très clairement, l'aléa est largement dominant dans cette approche, même si on prétend, de plus en plus, donner à la vulnérabilité une importance plus grande. La plupart des travaux qui se réclament de la problématique des risques sont en fait des études de l'aléa. Les atlas des risques sont le plus souvent des atlas des aléas. Toute approche des risques d'origine naturelle, en milieu urbain ou non, est fondamentalement "aléa centrée " (Cartier, Vinet et Gaillard, 2009) c'est à dire basée sur l'exposition d'un territoire à un ou plusieurs aléas (Coanus et al., 2010). En effet, que les études soient le fait d'administrations, dans la perspective de l'élaboration de plan de prévention des risques, ou de scientifiques pour produire des connaissances, l'aléa est toujours le point de départ des analyses. Et ce, non seulement pour les sciences de la terre et la géographie physique (qui, après tout, ont toute légitimité pour le faire vu leur objet), mais aussi pour la plupart des études de sciences sociales sur les risques. Dans la

EchoGéo, 18 | 2011 
plupart des analyses, l'aléa va non seulement orienter, déterminer toute la démarche, mais également délimiter l'espace qui est pertinent pour l'analyse du risque.

La logique générale initiale de cette approche "aléa x vulnérabilité » exprime l'idée qu'on a d'un coté un aléa actif, qui agit, qui fait le risque, qui produit des dommages, de la destruction, et de l'autre une vulnérabilité qui renvoie aux conséquences de l'aléa sur des éléments passifs. L'aléa se définit donc par une probabilité d'occurrence et une intensité d'un phénomène dommageable, et la vulnérabilité par l'estimation des dommages sur des supports passifs, les conséquences.

Malgré les controverses et évolutions autour de la notion de risque, le paradigme risque = aléa $\mathrm{x}$ vulnérabilité et la primauté de l'aléa restent au fondement de la très grande majorité des recherches (Gilbert, 2009). Ce paradigme continue à être amplement diffusé et solidifié par les méthodes d'élaboration des plans et politiques de prévention des risques, ancrant ainsi cette approche dans un large consensus technique. Il est également repris et reproduit dans la formulation des programmes de recherche scientifique.

L'évolution de la notion de risque principalement par l'évolution de la notion de vulnérabilité s'est faite sans remettre en cause le paradigme, ce qui met en évidence des problèmes conceptuels de fond :

- l'évolution de la notion de vulnérabilité vers la prise en compte d'une dimension plus active a fait perdre toute cohérence au paradigme " aléa x vulnérabilité "

- dans le paradigme « aléa x vulnérabilité », l'aléa et la vulnérabilité ne sont pas indépendants

- en milieu urbain notamment, l'aléa est incontestablement anthropisé

- la question de «ce qu'on peut perdre » qui est nécessairement centrale dans le risque n'a pas de statut conceptuel dans le paradigme, même si la question posée par les « éléments exposés » ou des « enjeux exposés » est de plus en plus présente.

Reprenons les différentes notions les unes après les autres.

\section{Aléa}

"L'aléa est un événement plus ou moins inattendu à l'origine de la catastrophe ", interprétationconfirmée par des classifications du type : "les phénomènes naturels créateurs de dommages (menaces) : diagnostic, inventaire et typologie » (Thouret et al., 1994). On parle le plus souvent de l'aléa comme d'un élément extérieur, indépendant de la vulnérabilité. Il est analysé par des physiciens comme un objet dont on reconnaît qu'il est plus ou moins (de moins en moins) naturel (Burton et al., 1978), sans qu'on trouve de véritable réflexion conceptuelle sur cette notion et sur sa place dans le risque. En fait, la notion ne fait pas ou peu discussion.

Or, en ville, l'aléa est le plus souvent anthropisé. On a une anthropisation indéniable des processus physiques appelés aléas, en particulier des risques d'inondation ou de crue qui sont les risques d'origine naturelle les plus courants, et ceux qui font le plus de dégâts dans les grandes villes du monde, en particulier dans les villes du sud. Les recherches mettent incontestablement en évidence des interactions entre aléa et vulnérabilité qui laissent à entendre qu'il ne s'agit pas de deux objets différents appréhendables séparément par des disciplines différentes.

Bien que le concept de risques soit largement discuté, le paradigme "aléa $\mathrm{x}$ vulnérabilité » est très peu remis en cause. En fait, le concept d'aléa est assez peu 
discuté, alors qu'en parallèle la vulnérabilité peine à être définie clairement, et fait l'objet de beaucoup de discussions, d'analyses, de propositions conceptuelles et méthodologiques. (Pigeon, 2005 ; Becerra et Pelletier, 2009).

\section{Vulnérabilité}

40 A coté de l'aléa, le concept de vulnérabilité reste la partie faible de la définition des risques (Gilbert, 2009). Il fait l'objet de discussions et d'évolutions qui ont permis de prendre en compte un certain nombre de réalités sociales, mais sa place reste plutôt marginale. Il faut dire que face à l'évidence des aléas, en particulier des aléas d'origine naturelle (séismes, éruptions volcaniques, inondations, glissements de terrains ...) portés par les certitudes et les outils des sciences de la terre, les interrogations et évolutions de la notion de vulnérabilité génèrent une certaine méfiance, une certaine incrédulité, et font que la vulnérabilité ne tient pas face aux certitudes de l'aléa. La vulnérabilité apparait comme un objet des sciences sociales, par définition contingent, pas très clair, que chacun approche à sa manière pour rendre compte de choses différentes, mais qui ont toutes à voir avec l'intervention du social dans le risque.

Dans le champ scientifique, on parle de vulnérabilité à propos de toutes sortes de choses, à toutes sortes d'échelles, et à propos d'éléments aussi bien physiques, matériels et sociotechniques qu'à propos d'éléments sociaux ou humains. Mais le plus souvent, ce qui est attendu des sciences sociales dans les analyses de risques, c'est qu'elles décryptent la "vulnérabilité de la population», les "perceptions» ou «représentations » des risques par la population (en fait, souvent les représentations de l'aléa) et son « comportement ».

42 Ceci dit, l'importance de la vulnérabilité dans la problématique des risques est maintenant reconnue (Gilbert, 2009). La notion de vulnérabilité a progressivement évolué pour mettre en évidence son caractère actif: la vulnérabilité n'est plus seulement une propension à subir des dommages mais intègre aussi une capacité à les provoquer, les modifier, une capacité à faire face à une catastrophe (résilience).... (D’Ercole, 1994 ; Blaikie et al., 1984 ; Pelling, 2003).

Dans la définition initiale du risque, on avait une position mineure et passive de la vulnérabilité qui venait appuyer la suprématie de l'aléa. Il y avait une relation conceptuelle logique entre aléa et vulnérabilité. Mais la vulnérabilité entendue comme une dimension sociale et active du risque, la vulnérabilité entendue comme quelque chose de l'ordre du risque produit par la société elle-même (Beck, 2001), cette nouvelle vulnérabilité maintenue à coté de la notion prépondérante d'aléa, au sein d'un paradigme resté inchangé, a rendu ce dernier en partie incohérent. (D'Ercole et Metzger, 2005).

44 Aujourd'hui quand on parle de vulnérabilité, on fait aussi bien référence à l'exposition à des aléas, qu'à une certaine fragilité ou susceptibilité d'endommagement. Pour rendre compte de la fameuse «dimension active » de la vulnérabilité, celle-ci exprime aussi une capacité à modifier voire à engendrer des aléas, une capacité à provoquer des catastrophes, une capacité à provoquer des crises (Blaikie et al., 1994). On peut aussi mettre dans la notion de vulnérabilité la capacité à faire face à ces catastrophes, à savoir faire face aux situations de crise, ce qui ferait de la vulnérabilité la résultante d'un bilan entre des fragilités et des capacités de résistance. On parle de vulnérabilité surtout à propos de la population, mais la vulnérabilité est aussi un caractère attribué à 
des bâtiments, des systèmes politiques économiques ou techniques, des infrastructures, des territoires etc. L'approche de la vulnérabilité se fait généralement par la mise à jour de «facteurs de vulnérabilité » qui à leur tour découpent le champ du social par les objets des disciplines des sciences sociales (vulnérabilité institutionnelle, politique, économique, sociale etc...) (D'Ercole, 1994), finalement de la même façon que les sciences dures ont découpé les risques en fonction de leurs propres objets scientifiques. Parmi les facteurs de vulnérabilité de la population, c'est la pauvreté et les différentes manières d'appréhender cette pauvreté qui est majoritairement soulignée.

Les risques urbains sont vus comme spécifiques du fait d'endommagements supérieurs en ville, c'est-à-dire finalement qu'il y a beaucoup plus d'éléments exposés aux aléas : évidemment la population en premier lieu. Mais les villes sont aussi le cœur localisé et matérialisé de systèmes économiques, politiques, institutionnels et logistiques, éléments essentiels non seulement $d u$ fonctionnement urbain mais des sociétés modernes en général. La question des risques urbains se pose donc aussi dans ces termes : ce qui affecte un espace particulier dans une ville peut impacter l'ensemble de l'agglomération, ce qui affecte une ville peut avoir des conséquences bien au-delà du territoire affecté.

\section{Enjeux}

Comme cela a été souligné plus haut, la question de "ce qu'on peut perdre " est nécessairement centrale dans le risque, si ce n'est dans l'approche scientifique, tout sûr dans le sens commun. Si on n'a rien à perdre, il n'y a pas de risque. On peut en toute simplicité dire que le risque c'est la possibilité de perdre ce à quoi on accorde de l'importance. Or «ce que l'on peut perdre " n'a pas de statut conceptuel dans le paradigme « aléa $\mathrm{x}$ vulnérabilité ».

47 Le défi est donc de rendre une cohérence à la fois conceptuelle et opérationnelle à la notion de risque. C'est aussi de considérer " ce qu'on peut perdre ", c'est-à-dire l'enjeu comme un objet autonome dans la problématique des risques, de le dégager des notions d'aléa et de vulnérabilité qui structurent le concept pour dissocier clairement ce qu'on peut perdre (les enjeux) de ce qui peut provoquer leur perte (la vulnérabilité).

La proposition que nous avons faite dans nos travaux à Quito (D'Ercole et Metzger, 2002, 2004, 2005) et qui sert d'assise conceptuelle au programme PACIVUR (D'Ercole et al., 2009), est de donner aux « enjeux », c'est-à-dire à " ce que l'on risque de perdre ", un véritable statut conceptuel, à hauteur de leur importance dans ce qui fait le risque, et à coté de la vulnérabilité. De cette façon, la recherche sur les risques peut détacher son regard focalisé sur la " menace ", c'est-à-dire sur « l'aléa », pour regarder de près et prendre au sérieux «ce qu'on risque de perdre ». C'est ensuite, dans un second temps, que l'on peut analyser comment et pourquoi on peut "perdre » ces éléments, c'est-àdire ce qui fait leur vulnérabilité.

49 Et dans ce «pourquoi et comment on risque de perdre quelque chose d'important » on a bien évidemment l'exposition aux aléas. Mais on a aussi d'autres choses. On se retrouve donc avec un risque qui ne se décompose plus entre aléa et vulnérabilité mais entre enjeux (à défaut d'autre terme) et vulnérabilité, la vulnérabilité comprenant l'exposition aux aléas.

50 On ouvre ainsi la possibilité de comprendre les risques sans aléas, ou comportant des aléas non identifiés. On se donne aussi les moyens d'analyser conjointement tous les 
aléas susceptibles d'affecter un enjeu, les interactions entre aléas, de dégager l'existence de risques en dehors de la présence d'un aléa, par exemple par la simple présence d'enjeux vulnérables. On se donne donc aussi la possibilité d'améliorer les politiques de prévention des risques en ouvrant clairement le champ de la prévention au-delà de l'évitement ou de la contention de l'aléa à laquelle elles se réduisent souvent du fait d'une vision du risque centrée sur l'aléa.

Dans cette optique, et plus particulièrement en milieu urbain, ce déplacement de l'objet peut bouleverser totalement la connaissance qu'on a des risques et la manière de s'en protéger. Car cette approche signifie que l'analyse du risque part de l'identification des enjeux majeurs d'un ensemble urbain, c'est-à-dire des éléments essentiels qui permettent le fonctionnement urbain qui pourraient faire l'objet de politiques de prévention prioritaires.

Dans le même temps, partir des enjeux (de ce que l'on peut perdre) met nécessairement en évidence le fait que ces derniers ne sont pas les mêmes en fonction du point de vue social et territorial, et de l'échelle à laquelle on se place, ce qui permet de rendre compte du sens commun et de la dimension sociale irréductible du risque. De plus, en partant des enjeux d'un territoire, la délimitation de l'espace objet d'étude des sciences sociales, et en particulier de la géographie des risques, n'est plus construite par des dimensions physiques et matérielles de l'aléa, mais bien par un objet social et territorial (Gleyze et Reghazza, 2007 ; Metzger et D'Ercole, 2009).

\section{Transmission de vulnérabilité}

On peut remarquer que dans la problématique des risques, les principaux objets et concepts circulent d'un statut à l'autre. L'aléa peut surgir d'une vulnérabilité, ce qui est vulnérabilité, vu d'un endroit, est aléa d'un autre point de vue, ce qui est enjeu est aléa, ce qui est aléa est vulnérabilité. La vulnérabilité d'un élément, d'un territoire fait la vulnérabilité d'un autre élément ou territoire, selon des circuits difficiles à reconstituer, tant cette circulation des objets du risque est intégrée aux systèmes sociaux et urbains (Lagadec, 2003; Michel-Kerjan, 2003; Offner, 2000; Gleyze et Reghazza, 2007). La vulnérabilité circule, se déplace, se transmet, en empruntant tous les liens possibles, matériels et immatériels entre objets matériels et immatériels... (Metzger et D'Ercole, 2009). Cette constatation permet de rendre compte de l'implication du " point de vue » dans la problématique des risques. On se trouve face à de la vraie complexité qui met sur la touche les approches trop étroitement déterministes. Le défi est d'ordonner tous ces éléments, de forger des concepts capables d'englober cette complexité, d'appréhender cette réalité, pour pouvoir agir et réduire la vulnérabilité des sociétés.

\section{Pour conclure : quelle légitimité des sciences sociales?}

La dimension sociale des risques est une évidence, elle est reconnue par tout le monde : même les sciences "dures" sont d'accord pour dire qu'il n'y a risque que si il y a société. C'est dans ce cadre là que les sciences dures, dans leurs projets de recherche, 
font appel aux sciences sociales, afin de "prendre en compte la dimension sociale des risques ». C'est une première étape.

Depuis la controverse entre Voltaire et Rousseau au XVIII ${ }^{e}$ siècle à propos du séisme de Lisbonne, depuis les travaux de U. Beck (2001) et l'ouvrage de Fabiani et Theys (1987) pour rester dans l'approche scientifique, au-delà de la dimension sociale du risque, c'est la construction sociale du risque qui reste à formaliser, à démontrer. Et cela ne pourra se faire que par la construction de concepts opératoires capables de rendre compte du réel, du monde social, et capable du coup de permettre une action efficace.

Les concepts ne sont pas des bulles pour complaire aux scientifiques, des mots qui flottent au dessus de la réalité. Ils agissent sur cette réalité parce qu'ils traduisent une compréhension du monde, expriment une formalisation et une compréhension des problèmes qui se posent à la société, qui permettent d'avoir prise sur les processus. La conception actuelle des risques en est la démonstration flagrante : la primauté de l'aléa dans cette conception impose objectivement une limitation à la compréhension des risques, et constitue de ce fait, une limitation de la capacité des politiques et actions publiques à diminuer la vulnérabilité des sociétés et des territoires.

Parallèlement, il faut bien le dire, les recherches en sciences sociales sur les risques ne sont pas à la hauteur des enjeux. Les sciences sociales manquent de stratégie conceptuelle. Généralement, elles ne cherchent pas à se dégager de la primauté de l'aléa, tant celui-ci est "évident». Les sciences de la terre sont en quelques sortes propriétaires de la problématique des risques d'origine naturelle. Les sciences sociales, dans la plupart des cas, construisent leurs problématiques à partir d'un aléa, les géographes prennent généralement pour objet les espaces délimités par les processus physiques, tels que compris par les sciences de la terre. L'objet risque des sciences sociales, en particulier en ce qui concerne les risques d'origine naturelle, est construit à partir des objets des sciences de la terre... il n'y a pas autonomie des problématiques sociales du risque, et c'est un problème de fond dans l'approche des risques, en particulier pour les risques d'origine naturelle en milieu urbain, et c'est aussi, selon nous, un problème de fond dans la légitimité de l'apport des sciences sociales à cette problématique.

58 Le défi pour les sciences sociales est de se distancier par rapport à l'aléa sans pour autant le reléguer comme quantité négligeable, ce qui aurait pour conséquence de marginaliser, voire de décrédibiliser, les sciences sociales auprès des acteurs des politiques de prévention des risques. C'est la tentative qui a été faite en proposant de définir le risque à partir des deux notions d'enjeux et de vulnérabilité. Dans ce cadre les " enjeux » sont définis en dehors de toute référence à l'aléa, par rapport à un territoire. Dans cette approche, l'exposition aux aléas est considérée comme une dimension de la vulnérabilité, parmi de nombreuses autres.

Il reste à réfléchir de plus près également, et dans les mêmes termes, c'est-à-dire en questionnant les concepts et leurs implications sociales, à la gouvernance des risques, à ce que recouvre la notion d'acceptabilité sociale des risques, aux liens entre environnement et risques, entre vulnérabilité et développement. 


\section{BIBLIOGRAPHIE}

Barraqué B., 1994. Risque d'inondation, urbanisme réglementaire et servitude, Espace et société $\mathrm{n}^{\circ}$ 77, Risques environnement, modernité, L'Harmattan, p. 133-152.

Becerra S., Peltier A., 2009. Risques et environnement : recherches interdisciplinaires sur la vulnérabilité des sociétés. Paris, l'Harmattan, 575 p.

Beck U., 2001. La société du risque, sur la voie d'une autre modernité. Flammarion, 521 p.

Blaikie P., Cannon T., Davis I., Wisner B., 1984. At risk. Natural people's vulnerability and disasters. London and New York, Routledge, $280 \mathrm{p}$.

Borraz O., 2005. Vers une sociologie du risque. In Risques crises et incertitudes : pour une analyse critique, Cahiers du GIS risques collectifs et situations de crise, p. 21-67.

Borraz O., 2008. Les politiques du risque. Presses de Sciences Po., 296 p.

Burton I., Kates R.W., White G.F., 1978. The environment as hazard. Oxford Univ. Press, New York, $240 \mathrm{p}$.

Callon M., Lascoumes P., Barthe Y., 2001. Agir dans un monde incertain. Éditions du Seuil.

Cartier S., Vinet F., Gaillard J.-C, 2009. Introduction : Maître du monde ou maître de soi. In Risques et environnement : recherches interdisciplinaires sur la vulnérabilité des sociétés, S. Becerra, A. Peltier (dir.), Paris, L'Harmattan, p. 10-20.

Coanus T, Pérouse J.-F., 2006. Villes et risques, Regard croisés sur quelques cités en danger. Paris, Economica, Anthropos, $235 \mathrm{p}$.

Coanus T., Comby J., Duchêne F., Martinais E., 2010. Risques et territoires. Interroger et comprendre la dimension locale de quelques risques contemporains. Lavoisier, Collection Sciences du risque et du danger, série Références, $480 \mathrm{p}$.

Dauphiné A., 2001. Risques et catastrophes. Observer, Spatialiser, Comprendre, Gérer. Collection U Géographie, Armand Collin, 288p.

De Terssac G., Gaillard I., 2008. La catastrophe d'AZF : l'apport des sciences humaines et sociales. Lavoisier, Collection Sciences du risque et du danger, série Références, 276 p.

D'Ercole R., Hardy S., Metzger P., Robert J., 2009. Vulnerabilidades urbanas en los países andinos (Bolivia, Ecuador, Perú). Bulletin de l'Institut Français d'Etudes Andines, 38 (3).

D'Ercole R., 1994. Les vulnérabilités des sociétés et des espaces urbanisés : concepts, typologie, modes d'analyse. Revue de Géographie Alpine, $\mathrm{n}^{\circ}$ 4, Tome LXXXII, p. 87-96.

D’Ercole R., Metzger P., 2009. Enjeux territoriaux et vulnérabilité, une approche opérationnelle. In Risques et environnement : recherches interdisciplinaires sur la vulnérabilité des sociétés, sous la direction de S. Becerra et A. Peltier, Paris, L'Harmattan, p. 391-402.

D'Ercole R., Metzger P., 2009. Las dimensiones espaciales del manejo de crisis : interés de la investigación y aplicación en Quito. In In D'Ercole R. (ed.), Hardy S. (ed.), Metzger P. (ed.), Robert J. (ed.). Vulnerabilidades urbanas en los países andinos (Bolivia, Ecuador, Perú). Bulletin de l'Institut Français d'Etudes Andines, 38 (3), p. 893-915. 
D’Ercole R., Metzger P., 2009. La vulnérabilité territoriale : une nouvelle approche des risques en milieu urbain. Cybergeo : European Journal of Geography, Vulnérabilités urbaines au sud, article 447, mis en ligne le 31 mars 2009, modifié le 14 mai 2009. URL : http://cybergeo.revues.org/22022

D’Ercole R., Metzger P., 2005. Repenser le concept de risque pour une gestion préventive du territoire, Rethinking the Concept of Risk for Preventive Land Management. Pangea, juindécembre 2005, p. 19-36.

D’Ercole R., Metzger P., 2004. Vulnerabilidad del Distrito Metropolitano de Quito. Colección Quito Metropolitano, MDMQ-IRD, Quito, Ecuador, 496 p.

D'Ercole R., Metzger P., 2004. Les enjeux au coeur de la définition du risques. Application à Quito (Equateur). In G. David (dir), Espaces tropicaux et risques. Du local au global, Actes des $\mathrm{X}^{\mathrm{e}}$ Journées de Géographie Tropicale, Presses Universitaires d'Orléans/IRD, p. 185-196.

D’Ercole R., Metzger P., 2002. Los lugares esenciales del Distrito Metropolitano de Quito. Colección Quito Metropolitano, MDMQ-IRD, Quito, Ecuador, 226 p.

Dourlens C., Vidal-Naquet P., 1992. La ville au risque de l'eau. Paris, L'Harmattan, 127 p.

Dubois-Maury J., C. Chaline, 2002. Les risques urbains. Paris, Armand Colin, 208 p.

Ewald F., 1997. Le retour du malin génie. In Godard O., Le principe de précaution, Paris, MSH /INRA, , p 99-126.

Fabiani J.- L.. Theys J., 1987. La Société vulnérable. Evaluer et maîtriser les risques. Paris, Presses de l'ENS, 1987.

Gilbert C., 1997. La précaution dans l'empire du milieu. In Godard O., Le principe de précaution, Paris, MSH/INRA, p. 311-330.

GILBERT C., 2007. Comment gérer des crises. Les pouvoirs publics face à des risques polymorphes. Regard sur l'actualité, l'Etat face aux risques, La Documentation Française, p. 61-78.

Gilbert C., 2009. La vulnérabilité, une notion vulnérable ? In Becerra S. , Peltier A. (dir.), Risques et environnement : recherches interdisciplinaires sur la vulnérabilité des sociétés, Paris, L'Harmattan.

Gleyze J.-F., Reghezza M., 2007. La vulnérabilité structurelle comme outil de compréhension des mécanismes d'endommagement, Géocarrefour, vol 82-1-2, p. 16-26.

Godard O. (dir), 1997. Le principe de précaution dans la conduite des affaires humaines. MSH Paris/ INRA, $351 \mathrm{p}$.

Godard O., Henry C., Lagadec P., E.Michel-Kerjan, 2002. Traité des nouveaux risques. Paris, Folio, $620 \mathrm{p}$.

Habermas J., 1973. La technique et la science comme idéologie. Paris, Gallimard, 211 p.

INRA CEMAGREF, 2008. Pesticides, agriculture et environnement, Réduire l'utilisation des pesticides et en limiter les impacts environnementaux, expertise collégiale INRA CEMAGREF. Quae, $120 \mathrm{p}$.

Jonas H., 1990. Le principe de responsabilité. Paris ,Editions du Cerf.

Lagadec P., 2003. Risques, crise et gouvernance : ruptures d'horizons, rupture de paradigme. Réalités industrielles, mai 2003.

Metzger P., 1999. Urban environment and risks: elements for discussion. In M.A. Fernandez (Compiler), Cities at Risk : environmental degradation, urban risk and disasters in Latin America, Quito, La Red/USAID, p. 59-76. 
Michel-Kerjan E., 2003. Risques catastrophiques et réseaux vitaux : de nouvelles vulnérabilités. Flux $n^{\circ}$ 51, p. 6-15.

Moriniaux V. (coord), 2003. Les Risques. Editions du Temps, 234 p.

Offner J.-M., 2000. Réseaux et dynamique urbaine. In Paquot T., Lussault M., Body-Gendrot S. (dir), La ville et l'urbain, l'état des savoirs, Paris, La Découverte.

Pelling M., 2003. The Vulnerabilities of cities: Natural disasters and social resilience. Londres, Earthscan, 256 p.

Pigeon P., 2005. Géographie critique des risques. Paris, Economica, Anthropos, 218 p.

Pigeon P., 2003. L'intérêt du risque pour l'enseignement de la géographie. In Moriniaux V., Les risques, p. 9-21.

Pigeon P., D'Ercole R., 1999. L'expertise internationale des risques dits naturels : intérêt géographique. Annales de Géographie, vol. 108 (608), p. 339-357.

UNDRO, 1986. Prévention et atténuation des catastrophes, vol. 11 : planification préalable. New York, Nations Unies, 242 p.

Theys J., Kaloara B. (dir.), 1992. La Terre outragée. Les experts sont formels! Autrement, 270 p.

Thouret J.-C. et al., 1994. Les phénomènes naturels créateurs de dommages : diagnostic, inventaire et typologie. Revue de Géographie Alpine, 82(4), p. 17-25.

Veyret Y (dir), 2003. Les risques. Paris, Sedes, 255 p.

\section{NOTES}

1. Les réflexions exposées ici sont alimentées tant par la bibliographie que par les recherches menées sur la vulnérabilité urbaine à Quito, à Lima et à La Paz ans le cadre du programme Pacivur (Programme andin de recherche et de formation sur la vulnérabilité urbaine), et du programme antérieur de recherche mené à Quito sous l'intitulé "Système d'information et risques dans le District Métropolitain de Quito». Pour plus de détails, voir nos travaux, en particulier : Los lugares esenciales del Distrito Metropolitano de Quito (2002) ; La vulnerabilidad del Distrito Metropolitano de Quito (2004) ; Repenser le concept de risque pour une gestion préventive du territoire (2005) ; Enjeux territoriaux et vulnérabilité, une approche opérationnelle (2009); La vulnérabilité territoriale : une nouvelle approche des risques en milieu urbain (2009).

2. Accident technologique majeur survenu à Toulouse en septembre 2001.

3. Voir en particulier les ouvrages de synthèse portant sur les risques : Dauphiné, 2001 ; Moriniaux, 2003 ; Veyret, 2003 ; Pigeon, 2005.

\section{RÉSUMÉS}

L'objectif de ce texte est de contribuer au débat sur la question des risques en milieu urbain, en reprenant les différentes manières d'aborder cette question, et en soulignant les problèmes conceptuels posés par l'approche des risques par les sciences sociales, notamment en géographie. 
Le texte tente de présenter les multiples points de vue et dimensions du risque, discute les différentes notions qui entrent dans sa définition, et argumente sur la nécessité de prendre en compte «ce qu'on risque de perdre » dans la définition du risque, ce qui renvoie d'une part à la construction sociale du risque, d'autre part à l'arbitrage entre risques opéré par les politiques publiques. Il insiste sur la nécessité pour les sciences sociales et la géographie de construire un concept dégagé de la primauté de l'aléa.

This text is a contribution to the debate on the question of the risks in urban zones. It exposes the various approaches of this question, and focuses on the conceptual problems put by the definition of the risks by the social sciences, in particular by the geography. The text exposes the multiple points of view and dimensions of the risk. It discusses the different notions which enter in its definition, and argues about the necessity of taking into account " what we can lose " in the definition of the risk. That position sends back on one hand to the social construction of the risk, on the other hand to the choice between the risks operated by the public policies. The text insists on the necessity for the social sciences and the geography to build a concept cleared of the superiority of the hazard.

El objetivo del texto es contribuir al debate sobre la cuestión de los riesgos en medio urbano, exponiendo las diferentes maneras de encarar esta cuestión y señalando los problemas conceptuales planteados por el enfoque de los riesgos en las ciencias sociales, en particular en la geografía. El texto presenta los múltiples puntos de vista y dimensiones del riesgo, cuestiona las nociones que entran en su definición y discute la necesidad de considerar "lo que se puede perder" en la definición del riesgo. Este planteamiento remite por un lado a la construcción social de los riesgos, por otro al arbitraje operado entre los riesgos por las políticas públicas. El texto insiste en la necesidad para las ciencias sociales y la geografía de construir un concepto soltado de la preeminencia del peligro.

\section{INDEX}

Mots-clés : risque, vulnérabilité, concept, politiques publiques, enjeux, sciences sociales, géographie

Palabras claves : riesgo, vulnerabilidad, concepto, políticas públicas, elementos esenciales, ciencias sociales, geografía

Keywords : risk, vulnerability, concept, public policies, stakes, social sciences, geography

\section{AUTEURS}

\section{PASCALE METZGER}

Pascale Metzger est chargée de recherches à l'IRD (programme PACIVUR - programme andin de formation et de recherche sur la vulnérabilité et les risques en milieu urbain) et membre de l'UMR Prodig. pascale.metzger@ird.fr. Elle a publié récemment :

- Metzger P., Couret D. et collectif Urbi, 2010. Vulnérabilité et pauvreté en milieu urbain. Réflexions à partir des villes du sud. In Coutard O. et Lévy J.-P. (dir), Ecologies Urbaines, Editions Economica, Anthropos, Collection Villes, p. 239-257.

- D’Ercole R. Metzger P., 2010. Différentiation spatiales et sociales, représentations et gestion du risque volcanique à Quito. In Coanus T., Comby J., Duchène F., Martinais E., Risques et territoires. Interroger et comprendre la dimension locale de quelques risques contemporains, Ed. Lavoisier, Collection Sciences du risque et du danger, série Références. p 193-206. 
- D'Ercole R., Hardy S., Metzger P., Robert J., 2009. Vulnérabilités urbaines dans les pays andins : introduction générale. In : D'Ercole R. (ed.), Hardy S. (ed.), Metzger P. (ed.), Robert J. (ed.), Vulnerabilidades urbanas en los países andinos (Bolivia, Ecuador, Peru). Bulletin de l'Institut Français d'Etudes Andines, 38 (3), p. 411-420.

http://www.ifeanet.org/publicaciones/detvol.php?codigo=456

\section{ROBERT D'ERCOLE}

Robert d'Ercole est directeur de recherches à l'Ird (programme PACIVUR - programme andin de formation et de recherche sur la vulnérabilité et les risques en milieu urbain) et membre de l'UMR Prodig. Robert.dercole@ird.fr. Il a publié récemment :

- D’Ercole R. Metzger P., 2010. Différentiation spatiales et sociales, représentations et gestion du risque volcanique à Quito. In Coanus T., Comby J., Duchène F., Martinais E., Risques et territoires. Interroger et comprendre la dimension locale de quelques risques contemporains, Ed. Lavoisier, Collection Sciences du risque et du danger, série Références. p 193-206.

- D'Ercole R., Hardy S., Metzger P., Robert J., 2009. Vulnérabilités urbaines dans les pays andins : introduction générale. In : D'Ercole R. (ed.), Hardy S. (ed.), Metzger P. (ed.), Robert J. (ed.), Vulnerabilidades urbanas en los países andinos (Bolivia, Ecuador, Peru). Bulletin de l'Institut Français d'Etudes Andines, 38 (3), p. 411-420.

http://www.ifeanet.org/publicaciones/detvol.php?codigo=456 